\title{
DOLOR Y TERMOGRAFÍA
}

\author{
David Alberto Rodríguez Medina \\ Facultad de Psicología, UNAM \\ México
}

\section{RESUMEN}

La valoración y manejo del dolor representa un desafío para el clínico tratante. Aun cuando las intervenciones psicológicas para el manejo del dolor han mostrado un sólido nivel de evidencia enmarcadas en las guías médicas, no existen guías psicológicas propias con algoritmos para la evaluación y tratamiento del paciente. En un esfuerzo bien conducido por las Dras. Flor y Turk (2011), se propuso el inventario multidimensional del dolor, mostrando cuatro perfiles de pacientes cuya principal diferencia fue la tensión muscular. Desde una perspectiva biopsicosocial puede considerarse el dolor crónico como un trastorno psicofisiológico. La presencia de dolor induce una respuesta nerviosa simpática, la cual influye en la respuesta inflamatoria. El objetivo de esta presentación es introducir al personal sanitario tratante sobre el uso de la imagen térmica infrarroja para la valoración de la actividad autonómica asociada al dolor como parte de un perfil biopsicosocial. Primero se aborda el tema de dolor y termografía, cuyos resultados muestran que, ante la presencia de dolor, la temperatura periférica (manos, nariz) decrementa. Los estudios piloto presentados sugieren que el manejo de estrategias de relajación incrementa la temperatura periférica e inducen la actividad parasimpática asociada al alivio. Finalmente se presenta la integración del perfil biopsicosocial del paciente con el propósito de desarrollar un plan de intervención y pronóstico afectivo con base en evidencia. En suma, se propone el uso de biomarcadores (citoquinas proinflamatorias, termobiomarcadores) como parámetro clínico para la evaluación e intervención psicológica, similar a los presentados por Flor y Turk (2011).
\end{abstract}

Palabras Clave:

Termografía, Dolor, Expresión Facial, IL-6

\section{PAIN AND THERMOGRAPHY}

\begin{abstract}
The assessment and management of pain represents a challenge for the treating clinician. Even though psychological interventions for pain management have shown a solid level of evidence framed in the medical guidelines, there are no psychological guides of their own with algorithms for the evaluation and treatment of the patient. In an effort well conducted by the researchs Flor \& Turk (2011), proposed the multidimensional inventory of pain, showing four profiles of patients whose main difference was muscle tension. From a biopsychosocial perspective, chronic pain can be considered a psychophysiological disorder. The presence of pain induces a sympathetic nervous response, which influences the inflammatory response. The objective of this presentation is to introduce the healthcare personnel on the use of infrared thermal image for the assessment of autonomic activity associated with pain as part of a biopsychosocial profile. First, the topic of pain and thermography is addressed, the results of which show that, in the presence of pain, the peripheral temperature (hands, nose) decreases. The pilot studies presented suggest that the management of relaxation strategies increases peripheral temperature and induces parasympathetic activity associated with relief. Finally, the integration of the biopsychosocial profile of the patient is presented with the purpose of developing an affective intervention and prognosis plan based on evidence. In short, the use of biomarkers (proinflammatory cytokines, thermobiomarkers) is proposed as a clinical parameter for psychological evaluation and intervention, similar to those presented by Flor \& Turk (2011).
\end{abstract}

\section{Keywords:}

Termography, Pain, Facial expression, IL-6

Bitácora del Artículo:

| Recibido: 1 de Febrero de 2018 | Aceptado: 1 Abril de 2018 | Publicado en línea: Enero-Junio de 2018 | 


\title{
Autoría y Derechos de Propiedad Intelectual
}

\section{DOLOR Y TERMOGRAFÍA}

\author{
David Alberto Rodríguez Medina
}

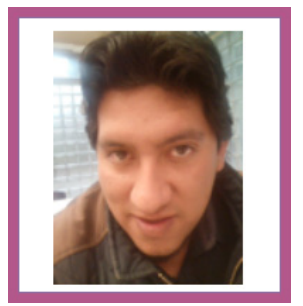

David Alberto Rodríguez Medina

Facultad de psicología, UNAM

Correo: psic.d.rodriguez@comunidad.unam.mx

Estudiante del programa de Doctorado en Psicología y Salud, Facultad de Psicología, UNAM. Diplomado en Psicofisiología Aplicada a la Psicología Clínica, INPRFM. Asesor clínico del servicio de Psicología del H. Ignacio Zaragoza, ISSSTE.

Ver más...

\section{PRESENTACIÓN DE LA CONFERENCIA}

La conferencia se presentó en el Segundo Curso Internacional de Nutrición y Prevención Cardiovascular e Insuficiencia Cardiaca y Respiratoria elaborado del 22 al 24 de noviembre de 2017 en el Instituto Nacional de Enfermedades Respiratorias Ismael Cosío Villegas

\section{AGRADECIMIENTOS}

A Gabriela Jael Pérez García, Viridiana Peláez Hernández, Benjamín Domínguez Trejo, Gerardo Leija Alva, Luis A. Morales Hernández e Irving A. Cruz Albarrán. | Investigación realizada con el financiamiento del proyecto académico: PAPIIT, Clave IN304515. Biomarcadores (autonómicos e inmunológicos) del componente emocional en el Dolor Crónico.

\section{DATOS DE Filiación del AUtor}

Facultad de psicología, UNAM.

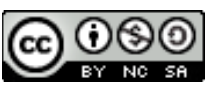

Copyright: (C) 2018 Rodríguez-Medina, D.A.

Este es un artículo de acceso abierto distribuido bajo los términos de la licencia Creative Commons Reconocimiento-NoComercial-Compartirlgual 4.0 Internacional, por lo que su contenido gráfico y escrito se puede compartir, copiar y redistribuir total o parcialmente sin necesidad de permiso expreso de su autor con la única condición de que no se puede usar con fines directamente comerciales y los términos legales de cualquier trabajo derivado deben ser los mismos que se expresan en la presente declaración. La única condición es que se cite la fuente con referencia a la Revista Digital Internacional de Psicología y Ciencia Social y a su autor. 


\section{TABLA DE CONTENIDO}

INTRODUCCIÓN

RELAJACIÓN Y TERMOGRAFÍA

INTEGRACIÓN DE LA ITF EN EL PERFIL BIOPSICOSOCIAL DEL DOLOR

CONCLUSIONES

REFERENCIAS 


\section{INTRODUCCIÓN}

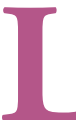

a capacidad clínica de reconocer una expresión facial emocional en residentes de la especialidad de Psiquiatría en México va incrementando conforme pasan sus años en la residencia (Arango, et al., 2014). Sin embargo, un reto clínico es valorar y determinar el nivel del dolor que experimenta un paciente. Por ejemplo, el diario estadounidense The New York Times (The New York Times, 2014) difundió los videos de un estudio (Bartlett, Littlewort, Frank \& Lee, 2014) para poner a prueba la precisión de reconocimiento facial del dolor de un software. Los resultados mostraron que el software superó las evaluaciones de un grupo de personas sin entrenamiento en reconocimiento de expresiones faciales. Sugerimos realizar el mismo reto con los videos que se muestran a continuación: se le mostrarán un par de proyecciones en la siguiente diapositiva. Su tarea es identificar en cuál de los dos videos (el de la izquierda o el de la derecha) la persona experimenta un dolor verdadero y cuál no lo es (Figura 1) (al finalizar la proyección de los videos se les solicita levantar la mano para votar la veracidad o falsedad de cada facie: el que se presenta del lado izquierdo de la pantalla es un video falso, mientras que el del lado derecho se trata de una facie de dolor verdadera).

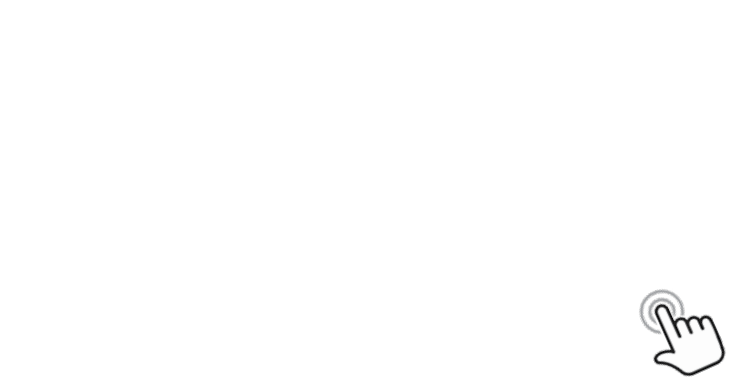

\section{Figura 1.}

Detección de expresión facial del dolor verdadera vs simulada (The New York Times, 2014)

Para conocer cuáles son los movimientos prototípicos de la expresión facial, particularmente la facie del dolor, se han evaluados las unidades de acción facial (AU) (Ekman \& Friesen, 1978). En una publicación de una de las revistas más influyentes en dolor, PAINC, se ilustran las AU de una facie de dolor (Chambers \& Mogil, 2015) (Figura 2). La presencia del dolor crónico modifica la capacidad de regulación de expresión facial (Vachon-Presseau et al., 2016)

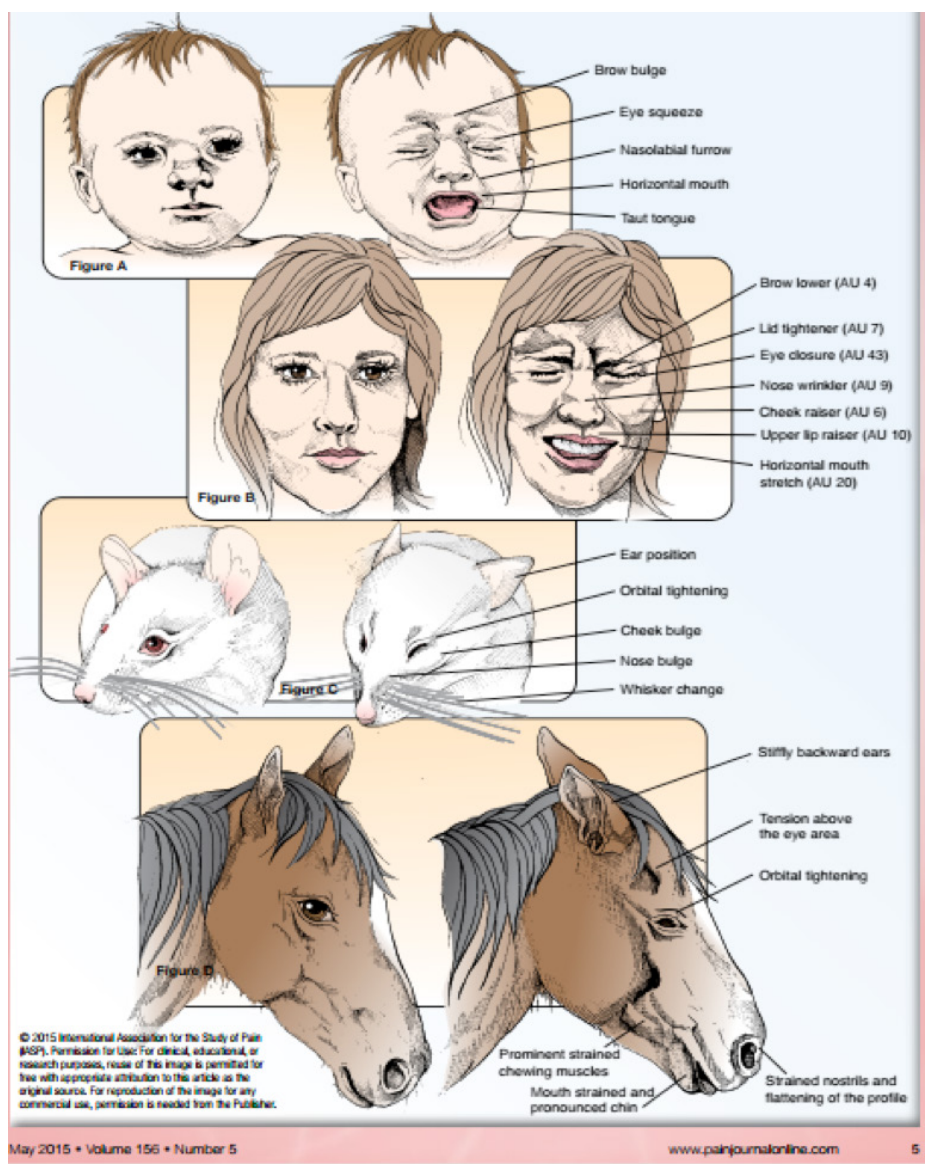

Figura 2.

Facie de dolor (Chambers \& Mogil, 2015).

Desde fines de la década de los 80s y hasta el presente se conoce que la expresión facial incrementa la actividad autonómica (Levenson, Ekman \& Friesen, 1990); en el caso del reconocimiento de la expresión facial de dolor también hay cambios psicofisiológicos cardiovasculaes (Jang, Park, Park, Kim \& Sohn, 2015). Esto posibilita que, si el personal de salud no cuenta con el entrenamiento en reconocimiento de microexpresiones faciales, se puede optar por una cámara de imagen térmica infrarroja (iTF), una herramienta portátil de registro psicofisiológico, útil para la valoración de actividad autonómica asociada al dolor. Para poder efectuar un protocolo de evaluación de imagenología térmica infrarroja se deben atender a una serie de consideraciones (Fernández-Cuevas et al., 2015).

La termografía tiene un uso potencial diagnóstico y tratamiento del dolor (Etehadtavakol, $\mathrm{Ng}, 2017)$. Entre sus aplicaciones clínicas en el campo del dolor se encuentran las enlistadas en la tabla 1 (Nahm, 2013). 
Tabla 1.

Indicaciones del uso de la iTF en la medicina del dolor

\begin{tabular}{|c|c|}
\hline Detección de Cáncer & Desórdenes de Dolor \\
\hline $\begin{array}{l}\text { - } \quad \text { Cáncer de piel } \\
\text { - } \quad \text { Cáncer de mama }\end{array}$ & $\begin{array}{l}\text { - } \quad \text { Síndrome complejo } \\
\text { - } \quad \text { Negional } \\
\text { - } \quad \text { Síndropatía periférica de dolor } \\
\text { miofascial } \\
\text { - Cefalea } \\
\text { - Lesión por latigazo } \\
\text { - } \quad \text { Nervical } \\
\text { - } \text { pouralgia } \\
\text { Artritis }\end{array}$ \\
\hline
\end{tabular}

Una de las revistas médicas latinoamericana especializada en el estudio de la actividad térmica (Pan American Journal of Medical Thermology) ha publicado el uso de la iTF como una herramienta útil al diagnóstico del dolor en un par de estudios más (Lima, Brioschi, Teixeira \& Neves, 2015; Trotta, \& Ulbricht, 2015), así como un par de guías para la evaluación térmica neuromuscular (American Academy of Thermology, 2015a), y las pautas para la termografía mamaria (American Academy of Thermology, 2015b).

Sin embargo, el aspecto afectivo (desagradable) del dolor se puede valorar mediante la actividad simpática asociada, como la temperatura periférica (manos, nariz). En la revisión de Ioannou, Gallese y Merla (2014) se muestra que el dolor decrementa la temperatura del rostro y las manos. Estudios recientes en iTF muestran que los estímulos desagradables decrementan la temperatura nasal. Por ejemplo, un estímulo auditivo desagradable como escuchar una pelea (Kano, Hirata, Deschner, Behringer \& Call, 2016) o visual mediante la exposición de una imagen (Kosonogov et al., 2017) (Figura 3).

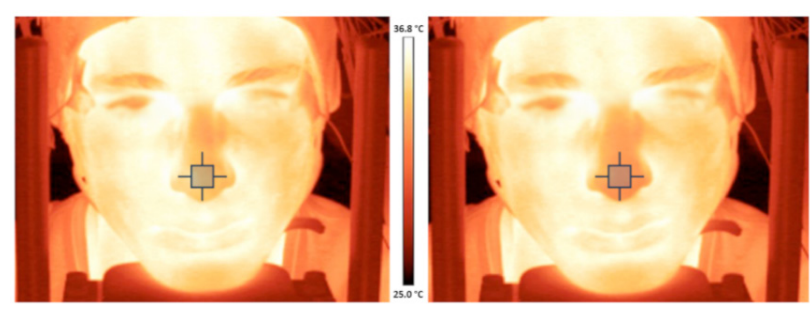

\section{Figura 3.}

Temperatura nasal. La imagen izquierda muestra al sujeto en reposo, mientras que la imagen derecha presenta el decremento de la temperatura nasal cuando se le proyectan imágenes desagradables (Kosonogov et al., 2017).
La actividad simpática propicia el estrechamiento de los vasos sanguíneos, o vasoconstricción, reduciendo el volumen sanguíneo lo que decrementa la temperatura periférica (y aumenta la presión sanguínea). Para contrarrestar esta actividad simpática existen estrategias que promueven la actividad parasimpática.

\section{Relajación y Termografía}

Un estudio publicado en una de las revistas más influyentes en ciencias, como Nature (Tyler et al., 2015), reportó estrategias para promover la actividad parasimpática: 1) ingesta de fármacos, 2) estrategias de relajación y 3) la estimulación transdermal (TEN), la cual es una placa ligera metálica colocada en la región del músculo corrugador para estimular nervios craneales y núcleos centrales que facilitan la actividad dorsovagal (asociada a estimulación parasimpática). Para evaluar la TEN utilizaron la imagen térmica (Figura 4), la cual muestra que la actividad parasimpática incrementa la temperatura facial.
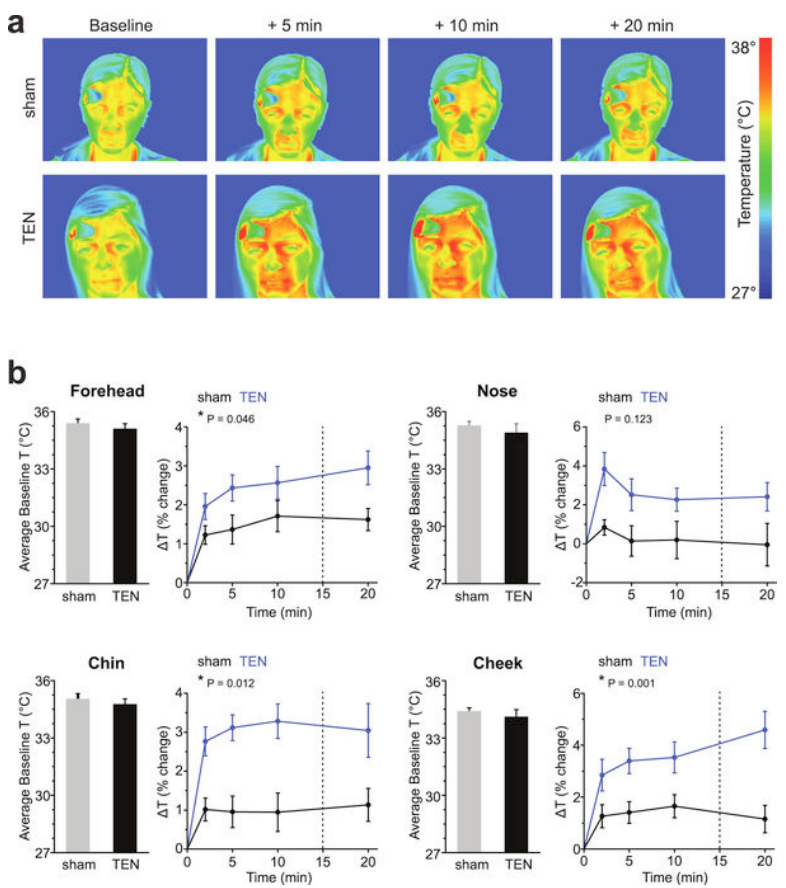

Figura 4.

Estimulación Transdermal para suprimir estrés psicofisiológico (Tyler et al., 2015).

¿Necesitaremos la TEN para promover la actividad parasimpática que disminuye el dolor? A nivel del SNC se ha señalado que podría disminuir la actividad cerebral que procesan el dolor inhibiendo la expresión facial (Kunz, Chen, Lautenbacher, Vachon-Presseau \& Rainville, 2011). Estudios recientes (Rodríguez-Medina, Domínguez, Cruz, 
Morales-Hernández \& Guzmán, 2017; Rodríguez-Medina, Domínguez, Cruz, Morales-Hernández, Leija \& Cortés, 2017) han evaluado el efecto de la relajación muscular facial y la respiración diafragmática. Dichos protocolos se han probado, primero, en participantes voluntarios $y$, posteriormente, en estudios piloto en población con dolor crónico oncológico y no oncológico. Los resultados son alentadores: se ha obtenido el mismo efecto, la relajación incrementa la temperatura en manos y nariz (Figura 5).

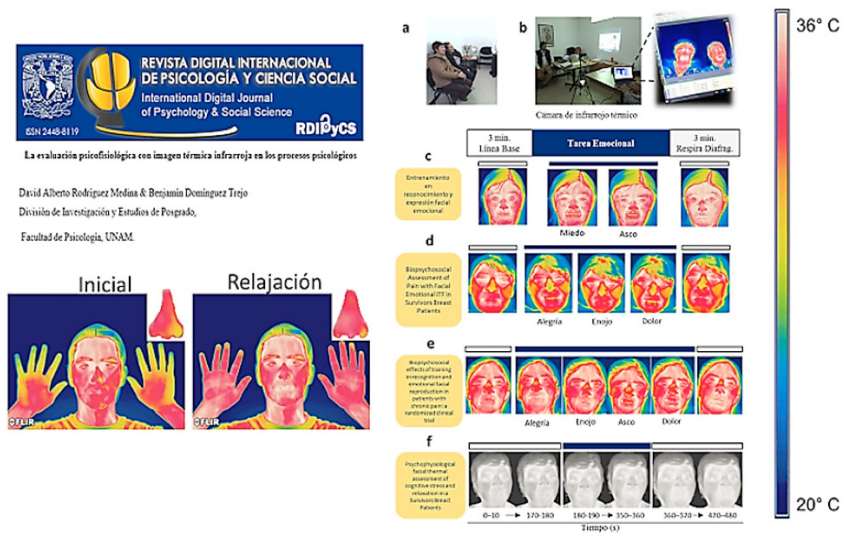

Figura 5.

Efectos de la expresión facial y la relajación sobre la temperatura periférica.

Lo que estos protocolos evalúan es lo que hemos llamado plasticidad térmica, que se refiere a la capacidad del sujeto en modificar su temperatura periférica. La expresión facial voluntaria (inducida con instrucciones de las AU durante al menos 5 segundos) genera los cambios autonómicos (Figura 6) (Rodríguez-Medina, Domínguez, Cruz, Morales-Hernández, \& Leija, datos no publicados). Si el participante cuenta con esta facilidad, su pronóstico afectivo es mejor a aquel que no logra relajarse.

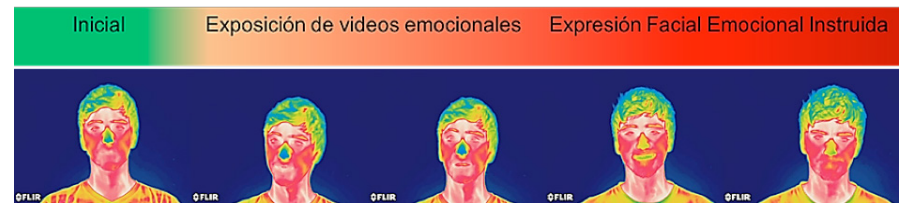

Figura 6.

Efecto de la expresión facial voluntaria sobre la temperatura nasal.

El estudio clínico que ilustra este efecto autonómico térmico se realizó en mujeres sobrevivientes de cáncer de mama (Rodríguez-Medina, Domínguez, Cruz, Morales-Hernández, Leija \& Cortés, 2018). El video de la Figura 7 (Ciencia UNAM, 2016) muestra el protocolo de evaluación e intervención.

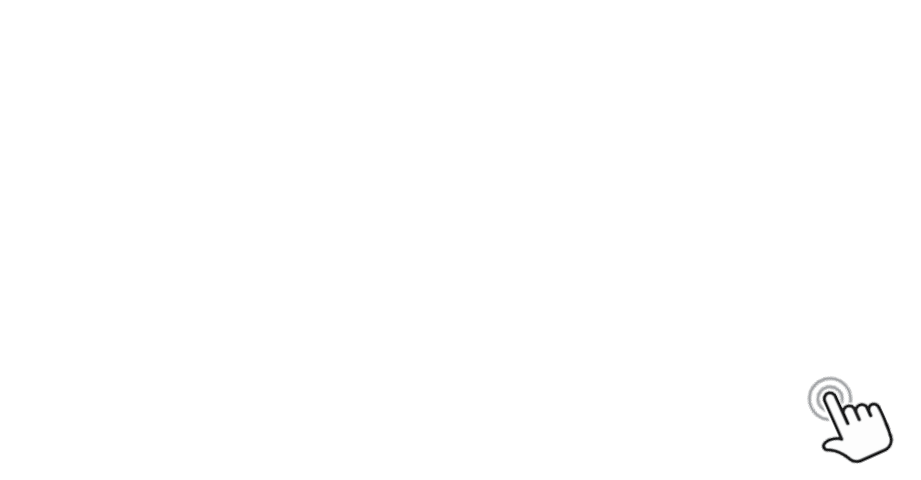

Figura 7.

Evaluación biopsicosocial del dolor crónico oncológico con imagen térmica

¿Cuáles fueron los resultados? La Figura 8 muestra el incremento de temperatura nasal y en manos de pacientes y controles. Hubo un efecto principal de la tarea, y en algunas zonas del rostro los cambios de temperatura fueron mayores en el grupo de sobrevivientes de cáncer de mama. Ello empata con su nivel apoyo social (superior al del grupo control) el cual facilita la interacción y promueve estados afectivos agradables y compañía para superar los obstáculos.
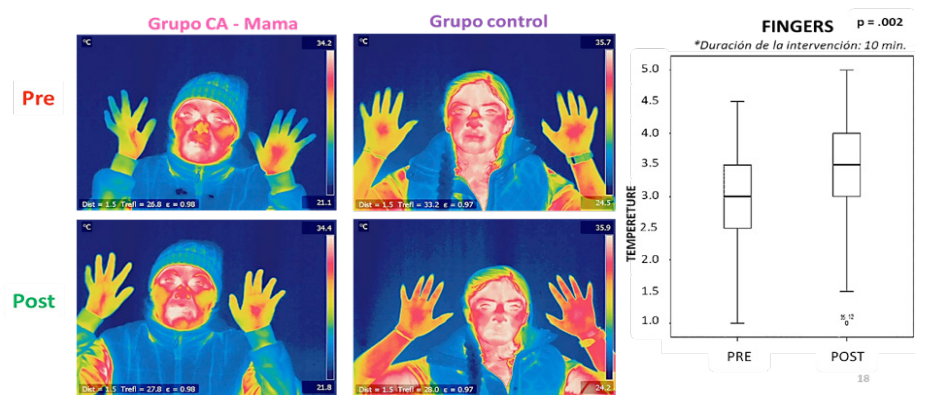

Figura 8.

Efecto de la exposición visual a estímulos agradables y relajación sobre la temperatura periférica

La capacidad de relajarse, en aproximadamente dos terceras partes del grupo de mujeres sobrevivientes de cáncer también se relacionó con su nivel de dolor: quien logró incrementar su temperatura periférica reportaron un menor nivel de dolor. Sin embargo, no todas lograron incrementar su temperatura, y esto estuvo asociado al nivel de la citoquina proinflamatoria IL-6 (Figura 9). Esto representa un desafío interdisciplinario para la investigación clínica que vamos a enfrentar en los próximos años. 


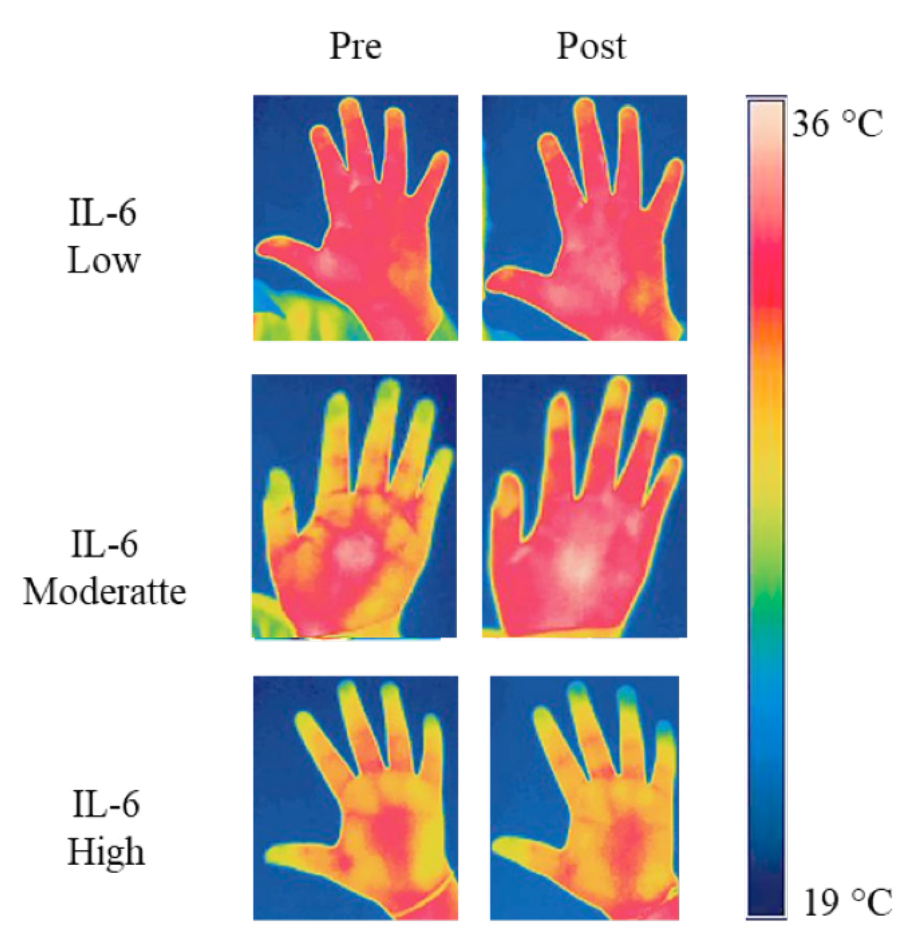

Figura 9.

Incrementos de temperatura en función del nivel de IL-6.

\section{INTEGRACIÓN DE LA ITF EN EL PERFIL BIOPSICO- SOCIAL DEL DOLOR}

La iTF brinda una valiosa información sobre la actividad autonómica. Para que el clínico pueda obtener un perfil de las necesidades de atención del paciente, es necesario integrarlas bajo el enfoque biopsicosocial. De esta manera se puede elaborar un plan de intervención particular e individual para el tratamiento psicológico de cada paciente. Para ilustrar la integración de la evaluación biopsicosocial se revisan dos casos de dolor crónico no oncológico y un caso de dolor oncológico. El primero, la evaluación biopsicosocial del paciente con diagnóstico de osteoartritis (Rodríguez-Medina, Cruz, Domínguez, Morales, Leija, \& Cortés, 2017) indicó un nivel considerable de interleucina 6 , con un nivel de dolor alto, interferencia conductual en varias áreas de su vida, con una cualificación del dolor predominantemente intermitente; niveles psicopatológicos moderados de ansiedad y depresión, pero con un buen control de ira y buena regulación emocional, aunque moderado apoyo social. Al realizar la evaluación termográfica de una tarea afectiva-cognitiva (primming afectivo) de sesgo atencional (es decir, un esfuerzo mental de discriminación afectiva negativa), se permite apreciar un decremento en la temperatura nasal durante la tarea afectiva (estrés) y su recuperación térmi- ca cuando el paciente respira diafragmáticamente (Figura 10), el cual concuerda con la frecuencia alta de su variabilidad de la frecuencia cardiaca. Los resultados sugieren que el paciente se beneficiaría de una intervención psicosocial que le permitiera desarrollar sus habilidades sociales, expresión emocional, y logros o desafíos personales, además de reforzar su capacidad de relajación.

\begin{tabular}{|c|c|}
\hline Dhneaskns of paln & Results \\
\hline \multicolumn{2}{|l|}{ Biological } \\
\hline - IL - 6 saliva sample & $8.6 \mu \mathrm{g} / \mathrm{ml}$ \\
\hline -HVR: High & $80 \%$ \\
\hline Low & $8 \%$ \\
\hline Very low & $12 \%$ \\
\hline \multicolumn{2}{|l|}{ Behavioral } \\
\hline - Number of Drugs for pain & 4 \\
\hline - Intensity of pain (NAS) & $8 / 10$ \\
\hline -Relief & $6 / 10$ \\
\hline - Behavioral interference & $8 / 10$ \\
\hline - Interference in your mood & 9/10 \\
\hline - Social interference & $8 / 10$ \\
\hline Qualities & Pts. \\
\hline -Continuous & 7 \\
\hline - Intermittent & 9 \\
\hline -Neuropathic & 4.5 \\
\hline -Affective & 5 \\
\hline Psychosocial & Pts. \\
\hline - Anxiety & 9 (moderate) \\
\hline - Depression & 11 (moderate) \\
\hline - External anger control & 15 (high) \\
\hline - Internal anger management & 12 (moderate) \\
\hline - Alexithymia & 22 (high) \\
\hline - Cognitive reevaluation & 32 (high) \\
\hline - Emotional suppression & 25 (high) \\
\hline - Social isolation & 59 (high) \\
\hline - Social support & 53 (moderate) \\
\hline Cognitive & Pts. \\
\hline - Loss or Damage & 10 (high) \\
\hline - Threat & 7 (moderate) \\
\hline -Challenge & 2 (low) \\
\hline
\end{tabular}

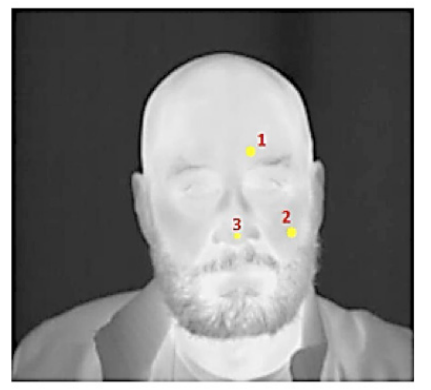

Figura 10.

Perfil biopsicosocial de un paciente con dolor crónico y entrenamiento en relajación.

La figura 11 muestra un ejemplo del perfil biopsicosocial del dolor oncológico. Utilizando escalas psicológicas validadas, se puede apreciar que la paciente sobreviviente de cáncer de mama presenta un dolor intenso, un bajo control de ira, un nivel moderado de ansiedad, depresión y apoyo social, así como un severo nivel de alexitimia (aspecto que está directamente relacionado al nivel del dolor). Precisamente la expresión facial y la relajación podrían serle de utilidad dentro de un programa de intervención psicológica especializado toda vez que su nivel de IL-6 es bajo y cuenta con plasticidad térmica. En suma, la paciente se beneficiaría mediante un programa de tratamiento integral (médico, nutricional y psicosocial). 


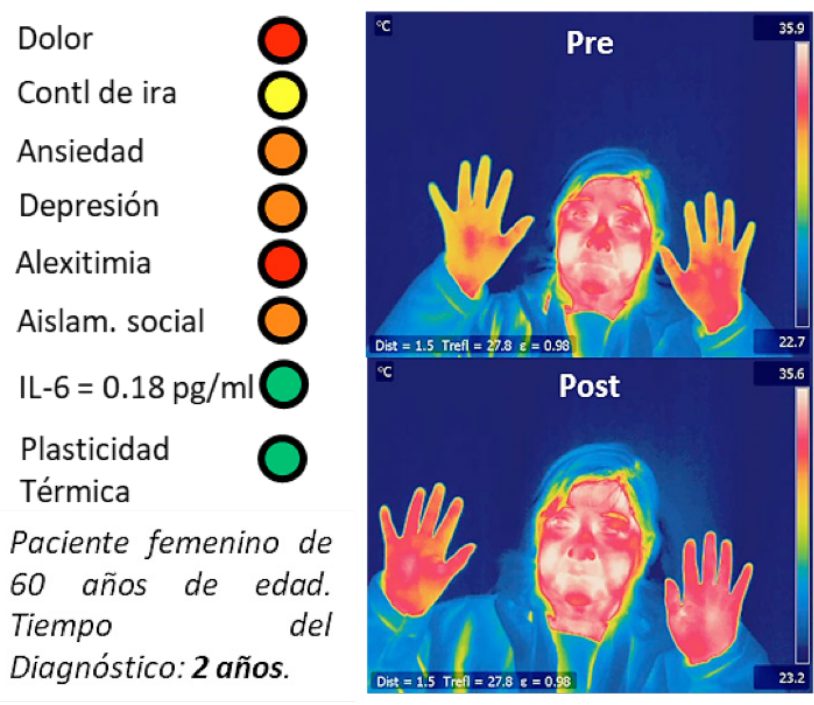

Peril Biopsicosocial.

Nivel óptimo $\mathrm{O}$ Nivel bajo $\mathrm{O}$ Nivel moderadoO Nivel alto $\mathrm{O}$

Figura 11.

Perfil Biopsicosocial del Dolor Crónico Oncológico.

\section{ConcLusiones}

La temperatura periférica puede considerarse un biomarcador de activación. Según el incremento o decremento de la temperaratura, se puede determinar la predominancia de la actividad autonómica (parasimpática o simpática, respectivamente). La imagen térmica infrarroja es sensible a estos cambios y supone una herramienta atractiva para el paciente al ser un equipo de registro fisiológico no invasivo.

\section{Referencias}

American Academy of Thermology (2015a). Guidelines for Neuromusculoskeletal Infrared Thermography Sympathetic Skin Response (SSR) Studies. Pan American Journal of Medical Thermology, [S.I.], 2(1), 35-43, http:// dx.doi.org/10.18073/2358-4696/pajmt.v2n1p35-43

American Academy of Thermology, A. (2015b). Guidelines for Breast Thermography. Pan American Journal Of Medical Thermology, 2(1), 26-34. http://dx.doi.org/10.18073/23584696/pajmt.v2n1p26-34

Arango de Montis, I., \& Fresán, A., \& Brüne, M., \& Ortega-Font, V., \& Villanueva-Valle, J., \& Saracco, R., \& Muñoz-Delgado, J. (2014). Evaluation of the capacity to recognize the facial expression of emotions in psychiatry residents throughout three years of training. Salud Mental, 37 (6), 455-460.

Bartlett, M., Littlewort, G., Frank, M., \& Lee, K. (2014). Automatic Decoding of Facial Movements Reveals Deceptive Pain Expressions. Current Biology, 24(7), 738-743. http:// dx.doi.org/10.1016/j.cub.2014.02.009
Ciencia UNAM. (2016). Proyecto Fénix [Image]. Retrieved from https://www.youtube.com/watch?v=FRWyel5WINw

Chambers, C., \& Mogil, J. (2015). Ontogeny and phylogeny of facial expression of pain. PAIN, 156(5), 798-799. http:// dx.doi.org/10.1097/j.pain.0000000000000133

Ekman P, Friesen W. (1978) Facial action coding system. Palo Alto, CA: Consulting Psychologists Press,

Etehadtavakol M., Ng E.Y.K. (2017) Potential of Thermography in Pain Diagnosing and Treatment Monitoring. En: $\mathrm{Ng}$ E., Etehadtavakol M. (eds) Application of Infrared to Biomedical Sciences. Series en BioEngineering. Springer, Singapore. https://doi.org/10.1007/978-981-10-3147-2_2

Fernández-Cuevas, I., Bouzas Marins, J., Arnáiz Lastras, J., Gómez Carmona, P., Piñonosa Cano, S., García-Concepción, M., \& Sillero-Quintana, M. (2015). Classification of factors influencing the use of infrared thermography in humans: A review. Infrared Physics \& Technology, 71, 28-55. http:// dx.doi.org/10.1016/j.infrared.2015.02.007

Flor, H., \& Turk, D. (2011). Identifying Patient Subgroups and Matching Patients with Treatments. In H. Flor \& D. Turk, Chronic Pain: An Integrated Biobehavioral Approach (1st ed., pp. 289-318). IASP press.

Ioannou, S., Gallese, V., \& Merla, A. (2014). Thermal infrared imaging in psychophysiology: Potentialities and limits. Psychophysiology, 51(10), 951-963. http://dx.doi. org/10.1111/psyp.12243

Jang, E., Park, B., Park, M., Kim, S., \& Sohn, J. (2015). Analysis of physiological signals for recognition of boredom, pain, and surprise emotions. Journal Of Physiological Anthropology, 34(1). http://dx.doi.org/10.1186/s40101-015-0063-5

Kano, F., Hirata, S., Deschner, T., Behringer, V., \& Call, J. (2016). Nasal temperature drop in response to a playback of conspecific fights in chimpanzees: A thermo-imaging study. Physiology \& Behavior, 155, 83-94. http://dx.doi. org/10.1016/j.physbeh.2015.11.029

Kosonogov, V., De Zorzi, L., Honoré, J., Martínez-Velázquez, E., Nandrino, J., Martinez-Selva, J., \& Sequeira, H. (2017). Facial thermal variations: A new marker of emotional arousal. PLOS ONE, 12(9), e0183592. http://dx.doi.org/10.1371/ journal.pone. 0183592

Kunz, M., Chen, J., Lautenbacher, S., Vachon-Presseau, E. \& Rainville, P. (2011). Cerebral Regulation of Facial Expressions of Pain. Journal Of Neuroscience, 31(24), 87308738. http://dx.doi.org/10.1523/jneurosci.0217-11.2011

Levenson, R., Ekman, P., \& Friesen, W. (1990). Voluntary Facial Action Generates Emotion-Specific Autonomic Nervous System Activity. Psychophysiology, 27(4), 363-384. http:// dx.doi.org/10.1111/j.1469-8986.1990.tb02330.x

Lima, R., Brioschi, M., Teixeira, M., \& Neves, E. (2015). Análise Termográfica de Corpo Inteiro: indicações para investigação de dores crônicas e diagnóstico complementar de disfunções secundárias. Pan American Journal Of Medical Thermology, 2(2), 70-77. http:// dx.doi:10.18073/2358-4696/pajmt.v2n2p70-77

Nahm, F. (2013). Infrared Thermography in Pain Medicine. The Korean Journal Of Pain, 26(3), 219. http://dx.doi. org/10.3344/kjp.2013.26.3.219

Rodríguez-Medina, D., Domínguez, B., Cruz, I., MoralesHernández, L., \& Guzmán, V. (2017). Termografía 
aplicada a la psicología de la salud. Revista De Psicología De La Universidad Católica De Santa María, 13, 7688. Retrieved from https://www.researchgate.net/ publication/321746931_TERMOGRAFIA_APLICADA_A PSICOLOGIA_DE_LA_SALUD_UTILIDADES_RETOS_Y ESTUDIO DE CASO

Rodríguez Medina, D., Domínguez Trejo, B., Cortés Esteban, P., Cruz Albarrán, I., Morales Hernández, L., \& Leija Alva, G. (2018). Biopsychosocial Assessment of Pain with Thermal Imaging of Emotional Facial Expression in Breast Cancer Survivors. Medicines, 5(2), 30. http://dx.doi.org/10.3390/ medicines5020030

Rodriguez Medina, D., Cruz, I., Domínguez, B., Morales, L., Leija, G., \& Cortés, P. (2017). Psychophysiological facial thermal assessment of the relaxation in a patient with osteoarthrosis. Pan American Journal Of Medical Thermology, 3, 33-36. http://dx.doi:10.18073/2358-4696/ pajmt.v3n1p33-36

Vachon-Presseau, E., Roy, M., Woo, C., Kunz, M., Martel, M., \& Sullivan, M. et al. (2016). Multiple faces of pain. PAIN, 157(8), 1819-1830. http://dx.doi.org/10.1097/j. pain.0000000000000587
The New York Times. (2014). Are These People in Real Pain or Just Faking It?. Retrieved from https://www.nytimes.com/ interactive/2014/04/28/science/faking-pain.html

Trotta, J., \& Ulbricht, L. (2015). Termografia no Diagnóstico Complementar de Doenças Músculo Esqueléticas [Thermography in Complementary Diagnostic of Musculoskeletal Diseases]. Pan American Journal Of Medical Thermology, 2(1), 7-13. http://dx.doi. org/10.18073/2358-4696/pajmt.v2n1p7-13

Tyler, W., Boasso, A., Mortimore, H., Silva, R., Charlesworth, J., \& Marlin, M. et al. (2015). Transdermal neuromodulation of noradrenergic activity suppresses psychophysiological and biochemical stress responses in humans. Scientific Reports, 5(1). http://dx.doi.org/10.1038/srep13865 